\title{
The CD4/CD8 ratio in BAL fluid is highly variable in sarcoidosis
}

\author{
S.P. Kantrow*, K.C. Meyer**, P. Kidd*, G. Raghu*
}

The CD4/CD8 ratio in BAL fluid is highly variable in sarcoidosis. S.P. Kantrow, K.C. Meyer, P. Kidd, G. Raghu. CERS Journals Ltd 1997.

ABSTRACT: Patients with pulmonary sarcoidosis frequently have increased numbers of lymphocytes and a high ratio of CD4+ to CD8+ T-lymphocytes (CD4/CD8 ratio) in bronchoalveolar lavage (BAL) fluid. Some investigators have suggested that these parameters can be used to distinguish sarcoidosis from other types of interstitial lung disease with a high degree of reliability. However, we hypothesized that the BAL CD4/CD8 ratio measured during the initial diagnostic evaluation of patients with biopsy-proven sarcoidosis is highly variable.

BAL lymphocytes were analysed via flow cytometry to determine the CD4/CD8 ratio in a population of 86 patients with histological and clinical evidence of sarcoidosis, who underwent BAL as part of their initial diagnostic evaluation.

In these patients, the CD4/CD8 ratio ranged $0.5-37.3$, with a median value of 3.35 (mean 6.49). The CD4/CD8 ratio was greater than 4 in only $36(42 \%)$ subjects. Ten patients $(12 \%)$ had a CD4/CD8 ratio less than 1 . The distribution of CD4/CD8 ratios was similar in the presence or absence of BAL lymphocytosis.

In conclusion, the CD4/CD8 ratio in bronchoalveolar lavage fluid is highly variable in biopsy-proven sarcoidosis. Bronchoalveolar lavage lymphocyte subset determination is a diagnostic test with low sensitivity for this disease. Eur Respir J 1997; 10: 2716-2721.
*Division of Pulmonary and Critical Care Medicine, Depts of Medicine and Laboratory Medicine, University of Washington Medical Center, Seattle, Washington, USA. **Division of Pulmonary and Critical Care Medicine, Dept of Medicine, University of Wisconsin, Madison, USA.

Correspondence: G. Raghu, Chief Chest Clinic, University of Washington Medical Center, Box 356522, Seattle, Washington, 98195-6522, USA

Keywords: Bronchoalveolar lavage, lymphocyte, sarcoidosis

Received: October 11996

Accepted after revision April 281997

This investigation was supported by NIH Research Training Grant HL07287 and funds administered by the University of Wisconsin Dept of Medicine, Medical School and Graduate School.
Sarcoidosis, a granulomatous disorder of unknown aetiology, commonly involves the lung and may involve other organ systems $[1,2]$. Affected individuals usually demonstrate hilar adenopathy, with or without pulmonary infiltrates on chest radiography. In addition, clinical involvement of the eye, skin and lymph nodes is frequently present in sarcoidosis. The pulmonary disease usually resolves spontaneously or stabilizes. Less commonly, pulmonary involvement is progressive and disabling [3]. The parenchymal inflammatory process is often treated with corticosteroids, although it responds inconsistently to therapy and may progress to endstage fibrosis.

The diagnosis of sarcoidosis is usually confirmed by the presence of noncaseating granulomas on transbronchial lung biopsy in the appropriate clinical setting [4]. Histological examination of other tissues, including conjunctiva, skin and lymph nodes, may also be diagnostic. Studies of bronchoalveolar lavage (BAL) fluid have demonstrated that sarcoid granuloma formation in the lung is often preceded by an influx of mononuclear cells into the alveoli. Activated alveolar macrophages and CD4+ (helper/inducer) T-lymphocytes dominate this early alveolitis [5-11]. These immunologically active cells release mediators, which appear to attract additional monocytes and induce formation of the characteristic epithelioid granulomas $[12,13]$. For this reason, the presence

For editorial comment see page 2699 of excess lymphocytes with a predominance of CD4+ T-lymphocytes in BAL fluid has been used as an adjunct to the clinical and histological assessment of the patient suspected to have sarcoidosis [14-17].

Patients with sarcoidosis may have an alveolitis in which the CD8+ (suppressor/cytotoxic) T-lymphocyte is the predominant T-cell type $[6,18,19]$. Agostini et al. [20] recently reported that $3.8 \%$ of sarcoid patients in their series had a BAL fluid CD4/CD8 ratio $<1$. They suggested that the clinical course does not differ substantially from that of patients with CD4+ T-lymphocyte predominant alveolitis. The appearance of CD8+ T-lymphocytes may represent usual progression of the local disease process, and may also be a precursor to granuloma development [21].

Several reports have suggested that analysis of BAL lymphocyte subsets to determine the ratio of CD4+ to CD8+ lymphocytes can be used to differentiate sarcoidosis from other causes of interstitial lung disease, including idiopathic pulmonary fibrosis and hypersensitivity pneumonitis [22-31]. The potential utility of this diagnostic approach depends upon a thorough knowledge of BAL cellular characteristics in sarcoidosis and competing interstitial lung diseases. We report our findings on BAL differential cell counts and lymphocyte subset ratios determined by flow-cytometry in a large group of patients with biopsy-proven sarcoidosis, who underwent BAL at the University of Wisconsin or at the University of Washington as a routine part of their initial evaluation. 


\section{Patients and methods}

The study population included 86 patients with sarcoidosis confirmed by biopsy between 1988 and 1993, who underwent BAL as part of their initial diagnostic evaluation at the University of Washington or the University of Wisconsin. Demographic data, radiographic stage, pulmonary function tests and BAL cellular profile were collected at the time of biopsy. The diagnosis of sarcoidosis was established by the presence of noncaseating granulomas in lung, mediastinal lymph nodes, skin or heart in patients with a compatible clinical history. Patients with evidence of malignancy, vasculitis or infection were excluded, as were those with a clinical history suggestive of berylliosis or hypersensitivity pneumonitis.

Fibreoptic bronchoscopy and BAL were performed as described previously $[32,33]$. Subjects were premedicated with atropine, and lidocaine was delivered topically via an atomizer. The bronchoscope was inserted transnasally or orally and passed to a subsegmental bronchus. BAL was performed in the right middle lobe or lingula if no focal changes were present on the chest radiograph. If localizing changes were present, BAL was performed in the area of greatest radiological abnormality. Adult nonsmoking normal volunteers were studied in accordance with human subject guidelines. Sterile isotonic saline at room temperature was instilled in five $30 \mathrm{~mL}$ aliquots (University of Washington) or four $60 \mathrm{~mL}$ aliquots (University of Wisconsin). Each aliquot was retrieved with gentle suction and pooled for subsequent cellular analysis. Despite variations in technique, cellular differential analyses of BAL are reproducible in normal volunteers using these lavage volumes [34, 35].

Analysis of the BAL fluid was performed as reported previously $[32,33]$. The BAL fluid was strained through a single layer of sterile gauze and centrifuged for $10 \mathrm{~min}$ at $400 \times \mathrm{g}$. The cell pellet was then resuspended in isotonic phosphate-buffered saline (PBS) and centrifuged for $5 \mathrm{~min}$ at $400 \times \mathrm{g}$. The supernatant was decanted and the cell pellet was resuspended in RPMI complete media (Gibco Laboratories, Grand Island, NY, USA) at the University of Washington or calcium- and magnesium-free Hank's balanced salt solution (HBSS) (Gibco Laboratories) at the University of Wisconsin. A cell viability count was performed by trypan blue exclusion. Cytocentrifuge preparations were made using a Shandon Cytospin 2 (Shandon Inc., Pittsburgh, PA, USA), and a $>200$ cell differential was performed on Wright's or Diff Quik stained slides. Aliquots of BAL cells were incubated at $4^{\circ} \mathrm{C}$ for $30 \mathrm{~min}$ with fluorescein isothiocyanate (FITC)-labelled anti-CD4 or anti-CD8 monoclonal antibodies, and washed once in buffer. The percentages of CD4 and CD8 positive cells were determined on a Coulter EPICS Profile flow cytometer (Coulter Cytometry, Hialeah, FL, USA) at the University of Washington or a FACSCAN or FACSTAR+ flow cytometer (BectonDickinson, Mountain View, CA, USA) at the University of Wisconsin. Lymphocytes were identified on the basis of dual parameter forward and side scatter, and files were gated to include only cells within the windows set on these parameters. The flow cytometry method correlates well with immunocytochemical determination of the $\mathrm{CD} 4 / \mathrm{CD} 8$ ratio across a wide range of total lymphocyte percentages [36].

Pulmonary function testing was performed routinely in each medical centre by registered pulmonary function technologists, in accordance with American Thoracic Society (ATS) recommendations. Interpretation of testing was based upon percentage of predicted values for spirometry, lung volume and transfer factor measurements. Biopsy specimens submitted for histology were evaluated by a pathologist unaware of the BAL data. Statistical comparisons of CD4/CD8 ratios between groups were carried out by nonparametric analysis, using the Mann-Whitney U-test [37].

\section{Results}

The group of sarcoidosis patients in this study was composed of an equal number of males and females. The subjects had a median age of 39 (range 18-78) yrs and $80 \%$ were Caucasian (table 1). The majority of patients presented for evaluation with either stage $1(36 \%)$ or stage $2(46 \%)$ radiographic abnormalities. Abnormalities of pulmonary function were detected in more than half of the 81 patients for whom comprehensive testing was available. A restrictive ventilatory defect was identified in approximately one quarter of the patients, and an obstructive defect in another quarter (table 1). Nineteen of the 23 patients with an obstructive defect had radiographic stage 2 or 3 disease, and 20 of the 23 patients with a restrictive defect had stage 2 or 3 disease. Of the 86 patients, 71 were nonsmokers, 11 were smokers, and four had an unknown history of cigarette use. Fourteen patients were receiving corticosteroid therapy at the time of evaluation.

Differential cellular analysis of the BAL fluid demonstrated lymphocytosis (lymphocyte count $>15 \%$ of the total nucleated cell count) in only $56(65 \%)$ of the study

Table 1. - Characteristics of the sarcoid patients $(n=86)$

\begin{tabular}{lrr}
\hline & $\mathrm{n}$ & $\%$ \\
\hline Age & & \\
$\quad<40$ yrs & 45 & 52 \\
$\quad \geq 40$ yrs & 41 & 48 \\
Sex & & \\
$\quad$ Male & 43 & 50 \\
$\quad$ Female & 43 & 50 \\
Race & & \\
$\quad$ Caucasian & 69 & 80 \\
$\quad 14$ & 16 \\
Other & 3 & 4 \\
Radiographic stage & & \\
0 & 3 & 4 \\
1 & 31 & 36 \\
2 & 40 & 46 \\
3 & 12 & 14 \\
Pulmonary function test results* & 36 & 44 \\
$\quad$ Normal & 19 & 23 \\
Restrictive only (TLC <80\% pred) & 19 & 5 \\
Obstructive only (ratio <0.70) & 4 & 4 \\
$\quad$ Restrictive and obstructive & 3 & \\
Low TL,CO only (<70\% pred) & & \\
\hline
\end{tabular}

*: spirometry, volumes and $T \mathrm{~L}, \mathrm{CO}$ were performed on 81 patients. TLC: total lung capacity; $\%$ pred: percentage of predicted value; TL,CO: transfer factor of the lung for carbon monoxide. 
Table 2. - Results of cellular differential analysis of bronchoalveolar lavage (BAL) from normal volunteers

\begin{tabular}{lllll}
\hline University $\mathrm{n}$ Macro Lymph Neutro Eosin CD4/CD8 \\
\hline
\end{tabular}

\begin{tabular}{lllllll}
\hline Washington & 35 & $82 \pm 10$ & $10 \pm 6$ & $4 \pm 6$ & $1 \pm 1$ & $1.49 \pm 0.59$
\end{tabular}

$\begin{array}{lllllll}\text { Wisconsin } & 34 & 89 \pm 7 & 10 \pm 6 & 1 \pm 1 & 0 \pm 1 & 1.53 \pm 0.80\end{array}$

Data are presented as mean \pm sD. Macro: macrophages; Lymph: lymphocytes; Neutro: neutrophils; Eosin: eosinophils.

population. In most patients, neutrophils accounted for $<1 \%$ of the BAL cells, but 20 patients $(23 \%)$ had $\geq 2 \%$ neutrophils. Similarly, most patients had $<1 \%$ eosinophils, but seven patients $(8 \%)$ had $\geq 2 \%$ eosinophils at the time of diagnosis. Normal values for BAL cellular analysis were determined from healthy nonsmoking volunteers, aged 19-47 yrs (table 2). An elevated percentage of neutrophils was observed in volunteers from the University of Washington, but lymphocyte percentage and lymphocyte subset ratios were similar at the two institutions. Nonparametric comparison of BAL findings from study patients and control subjects (University of Wisconsin) demonstrated that lymphocyte percentages and CD4/CD8 ratios were distributed differently in these two groups $(p=0.0002$ and $p \leq 0.0001$, respectively). The percentage of neutrophils and eosinophils did not differ significantly between study and control subjects.

The CD4/CD8 ratio was $>4.0$ in $36(42 \%)$ of the study population (fig. 1). The ratio was $2.5-4.0$ in $18(21 \%)$, and $1.0-2.5$ in $22(26 \%)$. Ten patients $(12 \%)$ had a CD4/ CD8 ratio of $<1$. The CD4/CD8 ratios were similarly distributed in patients with and without a BAL lymphocytosis (fig. 2). The BAL cellular profile of the patients with a low CD4/CD8 ratio was similar to the profile of patients with a normal or elevated CD4/CD8 ratio (table 3). Six patients had both BAL lymphocytosis and a CD4/ CD8 ratio of $<1$ (two of these were smokers).

The 14 patients who were receiving steroid therapy at the time of bronchoscopy had a mean lymphocyte percentage and median CD4/CD8 ratio which were similar to the overall population of sarcoid patients (21士 13 and $2.41 \pm 2.5 \%$, respectively). Three of these patients had a CD4/CD8 ratio of $<1$, and two of these had a BAL lymphocytosis. Because of the nonparametric distribution of the CD4/CD8 ratio, the Mann-Whitney

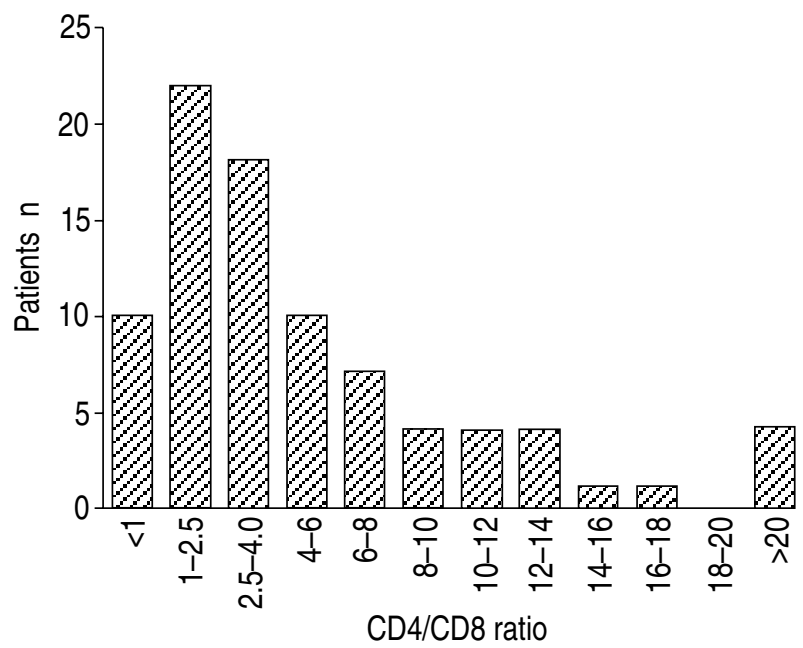

Fig. 1. - Distribution of bronchoalveolar lavage (BAL) CD4/CD8 ratios from patients with biopsy-proven sarcoidosis.

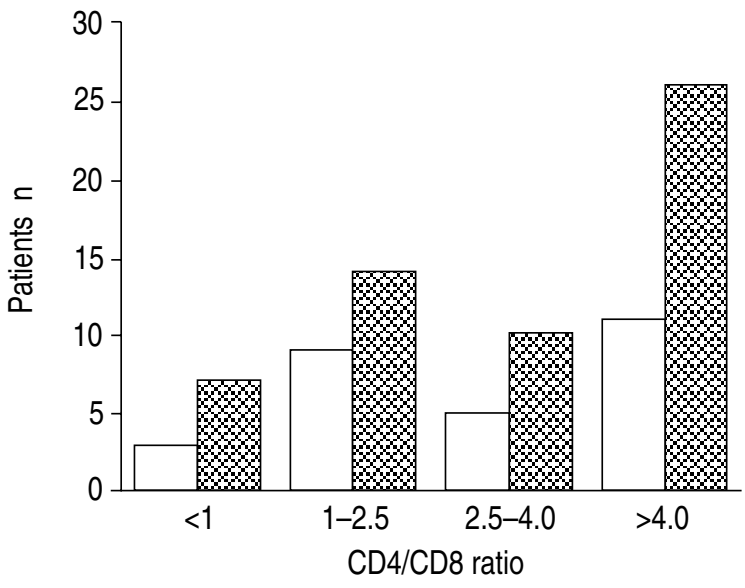

Fig. 2. - Distribution of bronchoalveolar lavage (BAL) CD4/CD8 ratios from patients with and without BAL lymphocytosis. $\square:<15 \%$ lymphocytes; $\mathbb{Q}: \geq 15 \%$ lymphocytes.

Table 3. - Results of cellular differential analysis of bronchoalveolar lavage (BAL) from patients with biopsyproven sarcoidosis

\begin{tabular}{lcccc}
\hline & Macro & Lymph & Neutro & Eosin \\
\hline All patients & $71 \pm 18$ & $25 \pm 17$ & $3 \pm 8$ & $0.5 \pm 1$ \\
& $(16-98)$ & $(1.6-78)$ & $(0-48)$ & $(0-10)$ \\
CD4/CD8 & & & & \\
ratio $<1$ & 78 & 19 & 1.8 & 0.2 \\
$(\mathrm{n}=10)$ & $(57-98)$ & $(1.6-43)$ & $(0-11)$ & $(0-1)$ \\
$\mathrm{CD} 4 / \mathrm{CD} 8$ & & & & \\
ratio $\geq 1$ & 70 & 25 & 3 & 0.5 \\
$(\mathrm{n}=76)$ & $(16-97)$ & $(2.3-78)$ & $(0-48)$ & $(0-10)$ \\
\hline
\end{tabular}

Data are presented as mean \pm SD percentage, and range in parenthesis. Macro: macrophages; Lymph: lymphocytes; Neutro: neutrophils; Eosin: eosinophils.

U-test was used to evaluate the influence of age $(<40$ versus $\geq 40 \mathrm{yrs}$ ), gender (male versus female), race (Caucasian versus African-American), and radiographic stage (1 versus 2 or 3 ). No significant correlation was found between these clinical variables and the CD4/CD8 ratio. To determine whether the CD4/CD8 ratio correlated with the serum level of angiotensin converting enzyme (ACE), an indirect marker of granuloma burden [38], the CD4/ $\mathrm{CD} 8$ ratios were plotted against the ACE level at time of diagnosis. Analysis of the 58 patients for whom the data were available demonstrated no correlation (linear curve fit, $r=0.14$ ).

\section{Discussion}

Cellular analysis of BAL fluid has enhanced our understanding of the pathophysiology of sarcoidosis in the lung. Recognition that lymphocytosis is common in BAL fluid from patients with sarcoidosis has suggested that sarcoidosis is a disease of heightened local immune cell activity [6]. Additional observations that CD4+ $\mathrm{T}$-cells are the predominant lymphocyte in BAL fluid have distinguished sarcoidosis from other disorders with BAL lymphocytosis, including hypersensitivity pneumonitis and lymphoma [31, 39, 40]. Studies of BAL cell products and their activity have suggested mechanisms of granuloma formation, and these observations have been fundamental to current concepts of the pathogenesis of sarcoidosis. Because we describe cell populations in BAL fluid collected only at the time of diagnosis, 
our data do not contradict previously suggested models of disease progression $[6,9,23]$.

The utility of BAL cellular analysis in the diagnosis of sarcoidosis is uncertain. Among 55 patients with interstitial lung disease and BAL lymphocytosis (27 with sarcoidosis), WinTERBAUER et al. [29] reported that a CD4/CD8 ratio (determined only on subjects with $>16 \%$ lymphocytes in BAL) greater than $4: 1$ had a positive predictive value of $94 \%$ for sarcoidosis. A CD4/CD8 ratio less than 1:1 excluded the diagnosis. The positive predictive value of the CD4/CD8 ratio fell to $50 \%$ when used to distinguish sarcoidosis from the entire group of 101 patients with BAL lymphocytosis and other types of lung disease including infections. Predictive values derived from this referral population of patients with parenchymal infiltrates and BAL lymphocytosis need to be confirmed in other centres. Importantly, BAL lymphocytosis is not a universal finding in sarcoidosis, as one third of the present patients with biopsy-proven sarcoidosis had $<16 \%$ lymphocytes in BAL.

DRENT et al. [30] applied discriminant analysis to their BAL experience (190 patients with sarcoidosis and radiographic stage 1 or 2 disease, 38 with extrinsic allergic alveolitis, and 44 with idiopathic pulmonary fibrosis). They found a positive predictive value of $92.2 \%$ and negative predictive value of $97.1 \%$ for BAL cell differential in diagnosing sarcoidosis, and then applied computerassisted analysis of BAL findings (excluding lymphocyte subset analysis) to a similar patient population with a high degree of accuracy [41]. CHECK et al. [26] generated likelihood ratios based upon relative percentage of lymphocytes in BAL from a database of 208 patients with BAL cellular differential analyses, and provided this information to clinicians evaluating patients for sarcoidosis.

If BAL cellular analysis is proposed as an adjunct to or replacement for transbronchial biopsy with histological evaluation in the diagnosis of sarcoidosis, the sensitivity of characteristic BAL findings must be examined. We found that approximately $40 \%$ of the present patients with biopsy-proven sarcoidosis had a CD4/ CD8 ratio greater than $4: 1$, consistent with the finding of Costabel et al. [42] that 52\% of 117 consecutive patients with biopsy-proven sarcoidosis had a CD4/CD8 ratio greater than 3.5. One potential clinical approach to the diagnosis of sarcoidosis is to proceed with $\mathrm{BAL}$ and cellular analysis without biopsy, using a CD4/CD8 ratio greater than 4 to confirm the diagnosis. In the present population, such an approach would have resulted in a nondiagnostic BAL (without biopsy) followed by a repeat bronchoscopy with biopsy in more than half of the cases. Our data provide no information concerning the utility of an elevated CD4/CD8 cell ratio in the setting of a negative transbronchial biopsy.

A CD4/CD 8 ratio of $<1$ was observed in $12 \%$ of the present biopsy-proven population of sarcoidosis patients. The CD8+ lymphocyte predominance is an unexplained finding in this disease. In the population studied, it occurred with and without lymphocytosis, and was not correlated with radiographic stage. Early reports of BAL and lung biopsy findings from patients with sarcoidosis suggested that the influx of CD4+ lymphocytes typically seen early in the alveolitis may be replaced by CD8+ lymphocytes as the disease stabilizes or be- comes inactive [4, 43]. Subsequent reports have not demonstrated an association of the CD4/CD8 ratio with duration of symptoms or radiographic stage [20, 44]. Our findings suggest that a low $\mathrm{CD} 4 / \mathrm{CD} 8$ ratio on $\mathrm{BAL}$ lymphocyte subset analysis should not be used to exclude the diagnosis of sarcoidosis.

The potential utility of the BAL CD4/CD8 ratio in the diagnosis of sarcoidosis depends upon several factors. Firstly, the frequency with which the BAL subset analysis yields a diagnostic (positive or negative) result should be high if centres employ this technique as a primary or supplementary diagnostic tool. In our experience, the CD4/CD8 ratio is suggestive of the diagnosis of sarcoidosis in less than $50 \%$ of biopsy-proven cases. Secondly, BAL findings associated with a high likelihood ratio for sarcoidosis (markedly elevated CD4/CD8 ratio) should contribute to the diagnosis. Some investigators have observed a strong correlation between the clinical presentation of sarcoidosis and an elevated CD4/ CD8 ratio $[4,45,46]$. In patients who have a chest radiograph compatible with sarcoidosis and evidence of acute inflammation (erythema nodosum or uveitis), the CD4/CD8 ratios tend to be substantially higher than in asymptomatic patients with sarcoidosis (mean ratio $\sim 10$ versus 3-5). Patients with this particular type of clinical presentation (bilateral hilar adenopathy with erythema nodosum or uveitis) have few competing diagnoses [47, 48], and the prognosis for symptomatic recovery is excellent [49]. Although results of BAL analysis in this subgroup of patients with sarcoidosis may prove both specific and sensitive, the contribution of cellular analysis to their diagnosis and management is uncertain. Finally, the predictive value of the CD4/CD8 ratio for sarcoidosis depends upon the clinical likelihood (prior probability) of sarcoidosis and the specificity and sensitivity of this BAL finding for the diagnosis. However, the cellular content of BAL is highly variable or poorly characterized for many lung pathologies, which may be difficult to distinguish clinically from sarcoidosis [50]. Factors such as race and radiographic stage can also influence BAL findings [11, 45, 51], and the CD4/CD8 ratio in BAL increases with age in normal subjects [52].

In summary, the use of bronchoalveolar lavage lymphocyte subset analysis in the diagnosis of sarcoidosis has a number of important limitations. In a large referral population, we found that an elevated CD4/CD8 ratio has a low sensitivity for sarcoidosis, and observed that this measurement may occasionally mislead the clinician. The distribution of CD4/CD8 ratios in patients with biopsy-proven sarcoidosis suggests that substitution of bronchoalveolar lavage cellular analysis for transbronchial biopsy is not advisable. As a diagnostic adjunct in cases of biopsy-negative sarcoidosis, its role remains poorly defined. Our experience does not support the routine clinical use of bronchoalveolar lavage cellular analysis to diagnose or exclude sarcoidosis.

\section{References}

1. Lynch JP, Strieter RM. Sarcoidosis. In: Lynch JP III, DeRemee RA, eds. Immunologically Mediated Pulmonary Diseases. Philadelphia, Lippincott, 1995; pp. 189216. 
2. James DG. Clinical picture of sarcoidosis. In: Schwartz MI, King TE, eds. Interstitial Lung Disease. St Louis, Mosby, 1993; pp. 159-178.

3. Hillerdal G, Nou E, Osterman K, Schmekel B. Sarcoidosis: epidemiology and prognosis. Am Rev Respir Dis 1984; 130: 29-32.

4. Verstraeten A, Demedts M, Verwilghen J, et al. Predictive value of bronchoalveolar lavage in pulmonary sarcoidosis. Chest 1990; 98: 560-567.

5. Rossman MD, Dauber JH, Daniele RP. Identification of activated T cells in sarcoidosis. Am Rev Respir Dis 1978; 117: 713-720.

6. Hunninghake GW, Crystal RG. Pulmonary sarcoidosis: a disorder mediated by excess helper T-lymphocyte activity at sites of disease activity. N Engl J Med 1981; 305: 429-434.

7. Crystal RG, Roberts WC, Hunninghake GW, Gadek JE, Fulmer JD, Line BR. Pulmonary sarcoidosis: a disease characterized and perpetuated by activated lung T-lymphocytes. Ann Intern Med 1981; 94: 73-94.

8. Schoenberger CI, Line BR, Keogh BA, Hunninghake GW, Crystal RG. Lung inflammation in sarcoidosis: comparison of serum angiotensin-converting enzyme levels with bronchoalveolar lavage and gallium-67 scanning assessment of the T-lymphocyte alveolitis. Thorax 1982; 37: 19-25.

9. Viale G, Codecasa L, Bulgheroni P, et al. T-cell subsets in sarcoidosis: an immunocytochemical investigation of blood, bronchoalveolar lavage fluid, and prescalene lymph nodes from eight patients. Hum Pathol 1986; 17: 476-481.

10. Rossi GA, Sacco O, Cosulich E, et al. Helper T-lymphocytes in pulmonary sarcoidosis. Am Rev Respir Dis 1986; 133: 1086-1090.

11. Muller-Quernheim J, Pfeifer S, Strausz J, Ferlinz R. Correlation of clinical and immunologic parameters of the inflammatory activity of pulmonary sarcoidosis. Am Rev Respir Dis 1991; 144: 1322-1329.

12. Gerli R, Darwish S, Broccucci L, Spinozzi F, Ramotti $P$. Helper inducer T-cells in the lung of sarcoidosis patients: analysis of their pathogenic and clinical significance. Chest 1989; 95: 811-816.

13. DuBois RM, Kirby M, Balbi B, Saltini C, Crystal RG. T-lymphocytes that accumulate in the lung in sarcoidosis have evidence of recent stimulation of the Tcell antigen receptor. Am Rev Respir Dis 1992; 145: 1205-1211.

14. Hunninghake GW, Bedell GN, Zavala DC, Monick M, Brady M. Role of interleukin-2 release by lung T-cells in active pulmonary sarcoidosis. Am Rev Respir Dis 1983; 128: 634-638.

15. Chretien J, Venet A, Danel C, Israel-Biet D, Sandron D, Arnoux A. Bronchoalveolar lavage in sarcoidosis. Respiration 1985; 48: 220-223.

16. Stoller JK, Rankin JA, Reynolds HY. The impact of bronchoalveolar cell analysis on clinicians' diagnostic reasoning about interstitial lung disease. Chest 1987; 92: 839-843.

17. Smith CM, Moser KM. Management for interstitial lung disease. Chest 1989; 95: 676-678.

18. Greening AP, Nunn P, Rudolf M, Rees ADM. Pulmonary sarcoidosis: alterations in bronchoalveolar lymphocytes and T-cell subsets. Thorax 1985; 40: 278-283.

19. Yamaguchi E, Haneda H, Okazaki N, Abe S, Kawakami Y, Nojima T. CD8 cell-dominant alveolitis in pulmonary sarcoidosis. Chest 1989; 95: 228-231.

20. Agostini C, Trentin L, Zambello R, et al. CD8 alveoli- tis in sarcoidosis: incidence, phenotypic characteristics, and clinical features. Am J Med 1993; 95: 466472.

21. Thomas PD, Hunninghake GW. Current concepts of the pathogenesis of sarcoidosis. Am Rev Respir Dis 1987; 135: 747-760.

22. Godard P, Clot J, Jonquet O, Bousquet J, Michel FB. Lymphocyte subpopulations in bronchoalveolar lavages of patients with sarcoidosis and hypersensitivity. Chest 1981; 80: 447-452.

23. Hunninghake GW, Kawanami O, Ferrans VJ, Young RC, Roberts WC, Crystal RG. Characterization of the inflammatory and immune effector cells in the lung parenchyma of patients with interstitial lung disease. Am Rev Respir Dis 1981; 123: 407-412.

24. Ginns LC, Goldenheim PD, Burton RC, et al. T-lymphocyte subsets in peripheral blood and lung lavage in idiopathic pulmonary fibrosis and sarcoidosis: analysis by monoclonal antibodies and flow cytometry. Clin Immunol Immunopathol 1982; 25: 11-20.

25. Cantin A, Begin R, Boileau R, Drapeau G, Rola-Pleszczynski M. Features of bronchoalveolar lavage differentiating hypersensitivity pneumonitis and pulmonary sarcoidosis at time of initial presentation. Clin Invest Med 1984; 7: 89-94.

26. Check IJ, Gowitt GT, Staton GW. Bronchoalveolar lavage cell differentiation in the diagnosis of sarcoid interstitial lung disease. Am J Pathol 1985; 84: 744-747.

27. Paradis IL, Dauber JH, Rabin BS. Lymphocyte phenotypes in bronchoalveolar lavage and lung tissue in sarcoidosis and idiopathic pulmonary fibrosis. Am Rev Respir Dis 1986; 133: 855-860.

28. Robinson BWS, Rose AH, Thompson PJ, Hey A. Comparison of bronchoalveolar lavage helper/suppressor Tcell ratios in sarcoidosis versus other interstitial lung diseases. Aust NZ J Med 1987; 17: 9-15.

29. Winterbauer RH, Lammert J, Selland M, Wu R, Corley D, Springmeyer SC. Bronchoalveolar lavage cell populations in the diagnosis of sarcoidosis. Chest 1993; 104: 352-361.

30. Drent M, Mulder PGH, Wagenaar S, Hoogsteden HC, van Velzen-Blad H, van den Bosch JMM. Differences in BAL fluid variables in interstitial lung diseases evaluated by discriminant analysis. Eur Respir J 1993; 6: 803-810.

31. Drent M, Wagenaar SS, Mulder PHG, van Velzen-Blad H, Diamant M, van den Bosch JMM. Bronchoalveolar lavage fluid profiles in sarcoidosis, tuberculosis, and non-Hodgkin's and Hodgkin's disease and evaluation of differences. Chest 1994; 105: 514-519.

32. Meyer K, Kaminski MJ, Calhoun WJ, Auerbach R. Studies of bronchoalveolar lavage (BAL) cells and fluids in pulmonary sarcoidosis. I. Enhanced capacity of BAL cells from patients with pulmonary sarcoidosis to induce angiogenesis in vivo. Am Rev Respir Dis 1989; 140: 1446-1449.

33. Siminski J, Kidd P, Phillips GD, Collins C, Raghu G. Reversed helper/suppressor T-lymphocyte ratio in bronchoalveolar lavage fluid from patients with breast cancer and Pneumoncystis carinii pneumonia. Am Rev Respir Dis 1991; 143: 437-440.

34. Daniele RP, Elias JA, Epstein PE, Rossman MD. Bronchoalveolar lavage: role in the pathogenesis, diagnosis, and management of interstitial lung disease. Ann Intern Med 1985; 102: 93-108.

35. The BAL Co-operative Group Steering Committee. Bronchoalveolar lavage constituents in healthy individuals, 
idiopathic fibrosis and selected comparison groups. Am Rev Respir Dis 1990; 141: S169-S196.

36. Dauber JH, Wagner M, Brunsvold S, Paradis IL, Ernst LA, Waggoner A. Flow cytometric analysis of lymphocyte phenotypes in bronchoalveolar lavage fluid: comparison of a two color technique with a standard immunoperoxidase assay. Am J Respir Cell Mol Biol 1992; 7: 531-541.

37. Merchant RK, Schwartz DA, Helmers RA, Dayton CS, Hunninghake GW. Bronchoalveolar lavage cellularity: the distribution in normal volunteers. Am Rev Respir Dis 1992; 146: 448-453.

38. Studdy PR, Lapworth R, Bird R. Angiotensin-converting enzyme and its clinical significance-a review. J Clin Pathol 1983; 36: 938-947.

39. Costabel U. The alveolitis of hypersensitivity pneumonitis. Eur Respir J 1988; 1: 5-9.

40. Semenzato G, Agostini C, Zambello R, et al. Lung Tcells in hypersensitivity pneumonitis: phenotypic and functional analyses. J Immunol 1986; 137: 1164 1172.

41. Drent M, van Nierop MA, Gerritsen FA, Wouters EF, Mulder PG. A computer program using BALF analysis results as a diagnostic tool in interstitial lung diseases. Am J Respir Crit Care Med 1996; 153: 736-741.

42. Costabel U, Zaiss AW, Guzman J. Sensitivity and specificity of BAL findings in sarcoidosis. Sarcoidosis 1992; 9 (Suppl. 1): 211-214.

43. Cueppens JL, Lacquet LM, Marien G, Demedts M, van den Eeckhout A, Stevens E. Alveolar T-cell subsets in pulmonary sarcoidosis: correlation with disease activity and effect of steroid treatment. Am Rev Respir Dis 1984; 129: 563-568.

44. Ainslie GM, Poulter LW, DuBois RM. Relation between immunocytological features of bronchoalveolar lavage fluid and clinical indices in sarcoidosis. Thorax 1989; 44: 501-509.

45. Ward K, O'Connor C, Odlum C, Fitzgerald MX. Prognostic value of bronchoalveolar lavage in sarcoidosis: the critical influence of disease presentation. Thorax 1989; 44: 6-12.

46. Drent M, van Velzen-Blad H, Diamant M, Hoogsteden HC, van den Bosch JMM. Relationship between presentation of sarcoidosis and T-lymphocyte profile: a study in bronchoalveolar lavage fluid. Chest 1993; 104: 795-800.

47. Israel HL. The diagnosis of sarcoidosis. Ann Intern Med 1968; 68: 1323-1326.

48. Winterbauer RH, Belic N, Moores KD. A clinical interpretation of bilateral hilar adenopathy. Ann Intern Med 1973; 78: 65-71.

49. Smellie H, Hoyle C. The natural history of pulmonary sarcoidosis. Q J Med 1960; 29: 359-358.

50. Davis GS. Bronchoalveolar lavage in interstitial lung disease. Semin Respir Crit Care Med 1994; 15: 37-60.

51. Mukae H, Kohno S, Morikawa T, Kusano S, Kadota J, Hara H. Two color analysis of lymphocyte subsets of bronchoalveolar lavage fluid and peripheral blood in Japanese patients with sarcoidosis. Chest 1994; 105: 1474-1480.

52. Meyer KC, Ershler W, Rosenthal NS, Lu X, Peterson $\mathrm{K}$. Immune dysregulation in the aging human lung. $\mathrm{Am}$ J Respir Crit Care Med 1996; 153: 1072-1079. 